\title{
Adrenal incidentaloma
}

G. Arnaldi, A.M. Masini, G. Giacchetti,

A. Taccaliti, E. Faloia and F. Mantero

\author{
Division of Endocrinology, Department of Internal Medicine, \\ University of Ancona, Ancona, Italy
}

\author{
Correspondence \\ F. Mantero \\ Clinica di Endocrinologia \\ Az. O spedaliera "U mberto I" \\ sede di Torrette \\ 60100 Ancona \\ Italia \\ Fax: + 39-071-887-300 \\ E-mail: clendo@popcsi.unian.it \\ Presented at the First \\ International M eeting on Adrenal \\ Disease: Basic and Clinical \\ Aspects, Ribeirão Preto, SP, Brazil, \\ August 31-September 2, 1999. \\ Research supported in part by \\ Associazione Italiana per la Ricerca Sul \\ Cancro (AIRC), and Ministero dell' \\ Università e della Ricerca Scientifica \\ e Tecnologica (MURST) \\ (No. 9806261488).
}

Received December 20, 1999 Accepted May 3, 2000

\section{Abstract}

Incidentally discovered adrenal masses, or adrenal incidentalomas, have become a common clinical problem owing to wide application of radiologic imaging techniques. This definition encompasses a heterogeneous spectrum of pathologic entities, including primary adrenocortical and medullary tumors, benign or malignant lesions, hormonally active or inactive lesions, metastases, and infections. Once an adrenal mass is detected, the clinician needs to address two crucial questions: is the mass malignant, and is it hormonally active? This article provides an overview of the diagnostic clinical approach and management of the adrenal incidentaloma. Mass size is the most reliable variable to distinguish benign and malignant adrenal masses. Adrenalectomy should be recommended for masses greater than 4.0 $\mathrm{cm}$ because of the increased risk of malignancy. Adrenal scintigraphy has proved useful in discriminating between benign and malignant lesions. Finally, fine-needle aspiration biopsy is an important tool in the evaluation of oncological patients and it may be useful in establishing the presence of metastatic disease. The majority of adrenal incidentalomas are non-hypersecretory cortical adenomas but an endocrine evaluation can lead to the identification of a significant number of cases with subclinical Cushing's syndrome (5-15\%), pheochromocytoma (1.5-13\%) and aldosteronoma (0-7\%). The first step of hormonal screening should include an overnight low dose dexamethasone suppression test, the measure of urinary catecholamines or metanephrines, serum potassium and, in hypertensive patients, upright plasma aldosterone/plasma renin activity ratio. Dehydroepiandrosterone sulfate measurement may show evidence of adrenal androgen excess.

\section{Introduction}

The term adrenal incidentaloma (AI) is a comprehensive definition for any adrenal mass discovered by noninvasive abdominal imaging techniques performed for reasons other than suspected adrenal disease; however, retrospectively, patients were often found to have had symptoms or signs related

\section{Key words}

- Adrenal incidentaloma

- Subclinical Cushing's syndrome

- Silent pheochromocytoma

- Aldosterone-producing adenoma 
nal masses varies in different series from 1 to $5 \%$ of patients imaged by CT (1-3). The wide range reflects the inclusion in some series of patients evaluated for staging or follow-up of extra-adrenal malignancy.

In any case, this rate is likely to be underestimated and it is probable that the prevalence of this "disease of modern technology" will continue to increase as a result of imaging advances (1). In fact, the incidence of adrenal nodules at autopsy has been reported in up to $32 \%$ of patients without pre mortem suspicion of adrenal disease (3-5).

The frequency of AI gradually increases with age: it is uncommon under 30 years and increases in middle and old age, peaking between the fifth and seventh decade. This finding could represent a compensatory growth in response to the local ischemic damage of arteriosclerotic disease or could merely reflect the higher number of diagnostic procedures performed on aged patients. Moreover, an increased frequency in patients with diabetes mellitus, hypertension and obesity has been described.

During the last few years, we had the opportunity to coordinate a very large multicentric study organized by the AI Study Group of the Italian Society of Endocrinology (AI-SIE) (6). The study included more than 1000 cases with a median age of 58 years and a significant prevalence of female patients (58\%). This figure confirms the re- sults of some large radiological series but could also be explained in part by a referral bias since in autopsy series no sex differences were found. Bilateral incidentalomas were discovered in $10 \%$ of cases according to literature data $(3,4)$.

\section{Differential diagnosis}

The differential diagnosis of this clinical problem includes Cushing's syndrome, pheochromocytoma, primary aldosteronism, primary and metastatic malignancy, myelolipoma, and non-hypersecretory cortical adenoma. In some cases, the incidental adrenal masses are infiltrative disease, fungal and tuberculosis infection, hemorrhage and lesions that masquerade as adrenal but arise from adjacent organs (e.g., kidney, pancreas, gallbladder, spleen, lymph nodes). The classification and the prevalence of different pathological conditions are shown in Tables 1 and 2 and Figure 1.

The optimal diagnostic and therapeutic approach to AI is still controversial. In any case, three important questions should be addressed: first of all, is there any chance for the mass to be malignant? Then, could it be hormonally active, even in the absence of a classical clinical picture? And important as well, is an extensive diagnostic work-up justified, in view of the discomfort and cost, in that particular patient?

Table 1 - Adrenal incidentaloma: classification.

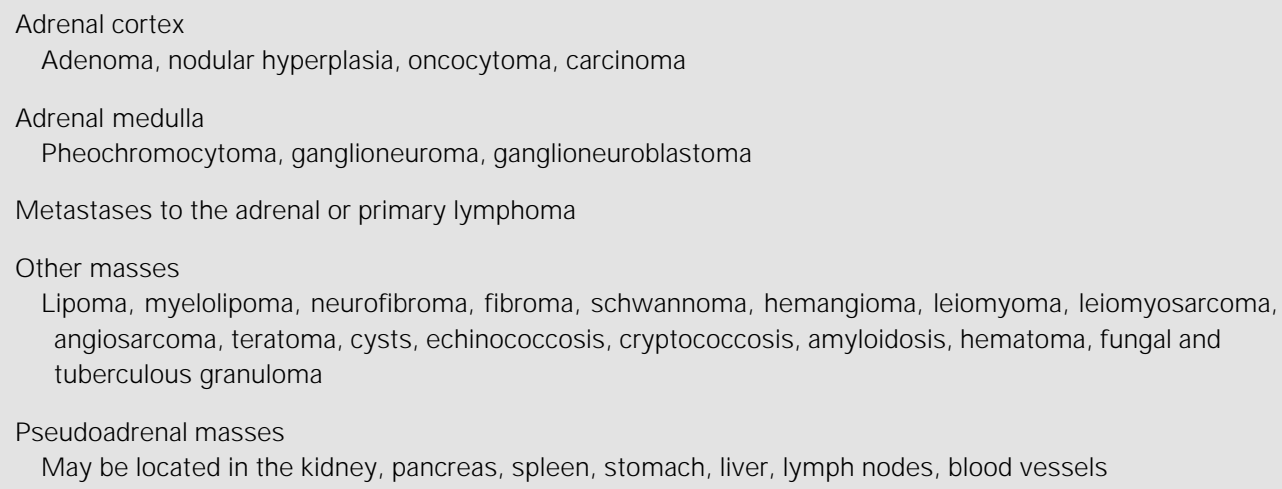




\section{Evaluation of malignancy}

Adrenocortical carcinoma is an uncommon neoplasm with an estimated annual incidence of $0.5-2$ cases per million population $(3,7)$. Most published series find a predominance of female patients (in some series up to $90 \%$ ). The prevalence of their functional status varies according to the series and the extension of the investigations performed, but in most cases they are nonhypersecretory masses. The clinical picture of carcinomas may be vague and nonspecific; these patients can in fact present with very mild signs of cortisol or androgen excess and/or nonspecific and slight abdominal and lumbar pain, or even be asymptomatic (8). For these reasons, adrenal carcinomas are usually diagnosed late in the course of the disease. Although the prognosis for localized disease is relatively good, with a 5year survival rate of $70 \%$, the rate plummets to $5.3 \%$ in the presence of metastases. Unfortunately, there are no clear circulating markers of malignancy, either in terms of classical tumor markers or endocrine param- eters, although in carcinomas an increase of 17-OHP (tumor dedifferentiation) or dehydroepiandrosterone sulfate (DHEAS) may elicit some concern (see below).

Presently, a significant number of primary adrenal carcinomas are detected incidentally and this finding may modify their

Table 2 - Prevalence of adrenal incidentaloma (AI).

Data are from the Study Group of the Italian Society of Endocrinology on Adrenal Incidentaloma (6).

\begin{tabular}{lc}
\hline & Al (\%) \\
\hline Non-hypersecretory adrenal adenomas & 74.0 \\
Hypersecretory tumors & \\
$\quad$ Cortisol-secreting adenomas & 9.2 \\
$\quad$ Aldosteronomas & 1.4 \\
Pheochromocytomas & 4.2 \\
Primary adrenal carcinomas & 4.0 \\
Other adrenal masses & \\
$\quad$ Myelolipomas & 3.0 \\
$\quad$ Cysts & 1.9 \\
Ganglioneuromas & 1.5 \\
Metastases & \\
$\quad$ Unselected patients & 0.7
\end{tabular}

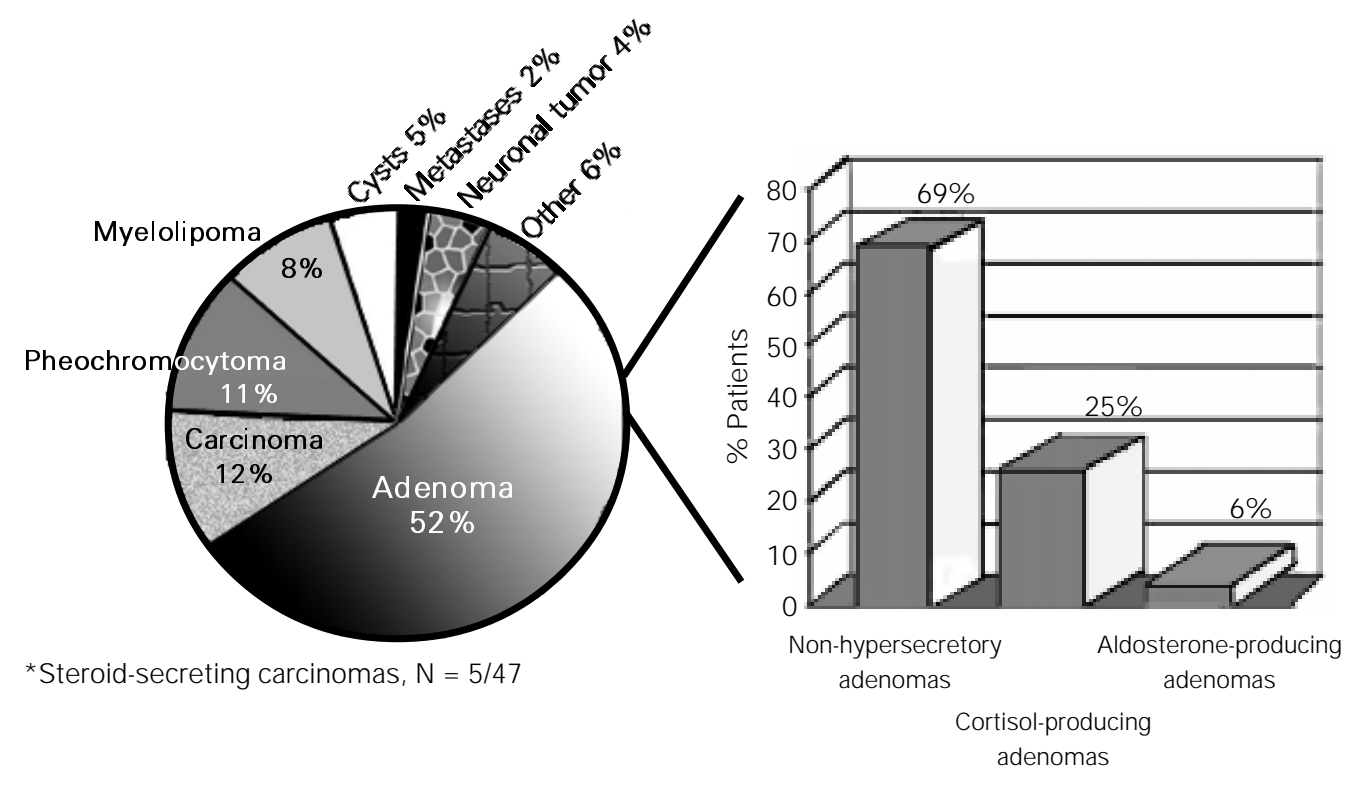

Figure 1 - Histological diagnosis of 376 adrenal incidentalomas. *From the results of the Study Group of the Italian Society of Endocrinology on Adrenal Incidentaloma (6). 
epidemiology. It is possible that the poor prognosis characteristic for this carcinoma may change into a better one with earlier detectability. The relative frequency of adrenal cancer varies considerably among AI series, ranging from 0 to $35 \%(3,7-9)$. In the largest series in the literature, comprising more than $1000 \mathrm{AI}, 47$ patients had a clinically silent primary adrenal carcinoma (4.6\%) (6). Abdominal pain was the most common reason leading to the discovery of carcinomas as a likely consequence of local infiltration, intratumoral hemorrhage or necrosis. Only 5 of these patients were producing a slight amount of steroids (cortisol and androgens); in 4 cases distant metastases were found at the time of the discovery of adrenal enlargement.

In the differential diagnosis, the lesion size is an important parameter of malignancy: the risk increases with increasing lesion diameter even though malignant tumors as small as $2.5 \mathrm{~cm}$ in diameter have occasionally been described.

The cut-off size for suspicion of malignancy is between 3 and $6 \mathrm{~cm}$. Based on the ROC curve for the diameter as calculated from data of the AI-SIE study (6), we consider $4 \mathrm{~cm}$ a reasonable cut-off value. Among the 47 cases of adrenal carcinoma found at surgery within the 387 patients of the AI-ISE study who underwent adrenalectomy, only 2 had a diameter lower than $4 \mathrm{~cm}$; the mean diameter was $7.5 \mathrm{~cm}$ and the range $2.6-25 \mathrm{~cm}$.

Although tumor size is highly predictive of malignancy, many types of adrenal lesions present as large masses, and in these cases additional information comes from other radiological features. The presence of a fatty adrenal mass is virtually diagnostic of myelolipoma, a usually non-functioning benign tumor composed of fat and normal bone marrow elements in varying proportions. However, atypical radiological features may occur in the presence of a high bone marrow/ fat ratio, hemorrhage or calcification in the tumor $(10,11)$.

Unfortunately, benign and malignant masses cannot be reliably discriminated on the basis of radiological features alone $(10,11)$. On CT scans, adrenocortical adenomas usually appear as small, homogeneous round masses with smooth margins, with relatively low density and without enhancement after intravenous contrast. The typical attenuation value ranges from -10 to 30 Hounsfield units (HU). By contrast, malignancies exhibit irregular margins and nonhomogeneous density with marked enhancement after intravenous contrast. At magnetic resonance imaging (MRI), malignancies usually show low signal intensity on T1-weighted images and high signal intensity on T2weighted images while most benign tumors have isointense or low signal intensity on both T1- and T2-weighted images. However, almost $30 \%$ of the masses cannot reliably be distinguished on T2-weighted images and again there is overlap between benign and malignant tumor appearance. Finally, after gadolinium administration adenomas show rapid contrast enhancement and rapid washout, whereas malignant lesions exhibit strong enhancement and slow washout.

The presence of necrosis, hemorrhage and calcifications is suspicious of malignancy, even though these aspects can also occur in large benign tumors. Both CT and MRI are accurate in assessing the spread of the tumor into tissues such as liver, lymph nodes, and lung. Moreover, the multiplanar capability of MRI is useful in demonstrating the invasion of the carcinoma, particularly into the inferior vena cava.

Other than in myelolipoma, in some pathologies such as pheochromocytoma, simple cyst, and adrenal hemorrhage, CT and MRI provide accurate tissue characterization. For instance, pheochromocytoma typically is isointense to liver on T1-weighted and hyperintense on T2-weighted images $(10,11)$. Whilst typical, these appearances on T2- 
weighted images are not specific. Indeed, both adrenal carcinoma and metastatic nodules may present with similar T2-weighted hyperintensity.

One recent, potentially promising technique is chemical-shift MRI, but its ultimate value in distinguishing benign from malignant masses has recently been shown to be disappointing $(10,11)$.

Metastases are a common cause of incidental adrenal masses, especially in oncological patients where the prevalence ranges from 32 to $73 \%$. Obviously, in these patients, distinguishing between metastases and other causes of adrenal enlargement may be critical in the management of primary cancer. Although the majority of metastases to the adrenal occur in the presence of metastases situated elsewhere, they may be the only site of tumoral spread. The adrenal glands are highly vascular organs and therefore common sites of metastases from extra-adrenal neoplasms, more frequently of the lung, breast, colon, kidney, or from melanoma or lymphoma. Adrenal metastases have been documented at autopsy in up to $38 \%$ of patients with cancer $(3,5)$.

However, when series of AI coming from Endocrine Divisions are evaluated, a much lower prevalence (less than $2 \%$ ) of adrenal metastasis is usually found in patients without a known extra-adrenal malignancy (6).

Adrenal metastases vary considerably in diameter. They tend to be smaller than primary adrenal cancer lesions and larger than adenomas.

For these reasons, size alone is a poor discriminator between adenomas and nonadenomas. The density of a mass as determined by measurement of the attenuation value on $\mathrm{CT}$ is a useful parameter for differentiating benign from malignant masses $(10,11)$. An adrenal adenoma contains lipids, whereas metastatic lesions do not, reflecting the histology of the primary neoplasm. A threshold of $10 \mathrm{HU}(18 \mathrm{HU}$ in some centers) and a threshold of $24 \mathrm{HU}$ with a 14- min delay on a contrast-enhanced CT scan, are used as the cut-off to distinguish between adenomas and metastases. Usually, metastatic lesions, often bilateral, have irregular margins, are not very homogeneous and show a thick, irregular enhancing rim after contrast.

If metastases are suspected, the ultrasound- or CT-guided fine-needle aspiration (FNA) biopsy is a useful diagnostic tool in the evaluation of adrenal lesions (sensitivity $80-95 \%$, specificity $99 \%$, accuracy $75-85 \%$ ) $(12,13)$. In our experience, the ultrasoundguided FNA not only has the advantage of being fast and done in real-time, without radiation exposure, but it also rarely misses its target.

FNA biopsy should be reserved for patients in whom the presence of adrenal metastasis may alter the therapy or prognosis, whereas FNA biopsy really has no proved efficacy in patients with AI and no history of malignancy. Unfortunately, FNA biopsy is seldom successful in distinguishing cortical adenoma from carcinoma. The potential hormonal activity of an AI must always be ascertained before performing an adrenal biopsy since this procedure could precipitate a potentially lethal hypertensive crisis in patients with pheochromocytoma.

Adrenal scintigraphy has proved useful in discriminating between benign and malignant lesions. Adrenal cortical scintigraphy using a radiocholesterol such as ${ }^{131} \mathrm{I}-6 \beta$ iodomethyl-19-norcholesterol (NP-59) or ${ }^{75} \mathrm{Se}$-selenomethylnorcholesterol, provides not only the anatomical localization of the adrenal glands, but also their in vivo functional characterization $(3,14)$.

A "discordant" scintigraphic pattern, i.e., demonstrating decreased or absent radiocholesterol uptake by the affected adrenal gland, is compatible with malignancy (primary and secondary) and other non-functioning space-occupying or destructive adrenal lesions. Conversely, unilateral adrenal visualization with virtual absence of the con- 
tralateral gland constitutes a "concordant" pattern almost typical of cortical benign adenoma. Nonetheless, well-differentiated carcinomas with radiotracer uptake have sometimes been described. "Concordant" uptake usually means that the adenoma accumulates the tracer (even though it does not process cholesterol to the final secretory products). The prevalence of this pattern in nonhypersecretory adenoma and in subclinical Cushing's syndrome (SCS) appears similar. Adrenal masses less than $2 \mathrm{~cm}$ in diameter yield an increased rate of non-lateralizing scans (i.e., a normal scan pattern demonstrating bilaterally symmetrical uptake) in patients with both malignant and benign lesions and represent the resolution limit of this technique $(15,16)$. Finally, a "discordant" scan can also point to a silent pheochromocytoma; in case of suspicion, scintigraphy with 131 or 123 I-m-iodobenzylguanidine ( ${ }^{131} \mathrm{I}-$ or $\left.{ }^{123} \mathrm{I}-\mathrm{MIBG}\right)$ should be performed.

A recent and more promising technique is the positron emission tomography (PET) with ${ }^{18} \mathrm{~F}$-fluorodeoxyglucose (FDG). Indeed, FDG-PET scanning correctly differentiated adenomas from adrenal metastases in 27 patients with bronchogenic carcinoma (17). However, this noninvasive technique showed a non-optimal specificity for malignancy.

\section{Evaluation of functional status}

Although in general the majority (67$94 \%$ ) of AI are non-hypersecretory adenomas, a hormonal screening evaluation can reveal a significant number of cases of clinically unsuspected hormone-secreting adrenal tumors $(2,4,6,18)$.

Glucocorticoid secretion and SCS. The most common incidental secreting mass appears to be the cortisol-secreting tumor (5$15 \%$ ). Indeed, a good proportion of patients with $\mathrm{AI}(50 \%)$ may have isolated or multiple slight abnormalities of the hypothalamo-pituitary-adrenal (HPA) axis such as high-bor- derline urinary free cortisol (UFC) excretion, impaired cortisol rhythm, only partial cortisol suppression with dexamethasone, low plasma $\mathrm{ACTH}$, or unresponsiveness to CRH (6). Adrenal insufficiency following surgical removal of "silent" adrenal adenomas was also described in $18-20 \%$ of cases and a partial suppression of the HPA axis can persist for several months after adrenalectomy.

These data suggest that subtle hypercortisolism may be a common finding in patients with incidental adrenal adenomas and this condition needs to be recognized in order to detect the possible appearance of an overt Cushing's syndrome. As the natural history of this condition is unclear, we agree with several authors who suggest that a better term for it is subclinical Cushing's syndrome rather than pre-Cushing (19).

However, the demonstration of this autonomous cortisol secretion is still a matter for debate (20). The uncertainty arises from the definition of incidentaloma which makes any definition of SCS based only on endocrine data somewhat arbitrary (21). The prevalence of SCS $(9.2 \%$ in the largest clinical study) varies greatly depending on the diagnostic criteria and the screening methods used $(3-5,18,20)$. Even though some authors suggest as an additional criterion the presence of a "concordant" uptake pattern on radiocholesterol scintiscan, this parameter is not universally accepted as it is found also in patients with an apparently normal HPA axis. Also in our experience the prevalence of "concordant" uptake is similar in both non-hypersecretory adenomas and in patients with SCS. To reduce the risk of a false-positive test and in the absence of a true gold standard test, we base our definition of SCS on at least two abnormal tests of HPA function in patients with AI and, by definition, without overt clinical stigmata of hypercortisolism.

In our personal series of $208 \mathrm{AI}, 29$ patients meet this stringent criterion with a 
$14 \%$ prevalence of SCS. The spectrum of cortisol excess in these patients may be wide: most have UFC of different degree; half may show an incomplete cortisol suppression $(>5$ $\mu \mathrm{g} / \mathrm{dl})$ after $1 \mathrm{mg}$ of dexamethasone overnight. The other hormonal abnormalities observed in these patients are loss of the normal diurnal rhythm of cortisol $(\sim 80 \%)$, low ACTH levels (50\%), and impaired ACTH response to the $\mathrm{CRH}$ test $(\sim 60 \%)$. The most frequent combinations encountered were unpaired cortisol suppressibility after overnight dexamethasone and above normal UFC excretion. Furthermore, isolated abnormal tests of the HPA axis are present in almost half of patients with incidentally discovered cortical adenoma $(6,20)$.

A long-term follow-up of patients with AI should be performed in order to evaluate the real significance of the mild endocrine alterations observed. The natural history of patients with AI is presently still unclear since the follow-up studies are limited to a small number of patients and to a short period of clinical evaluation. The majority of patients seem to maintain unchanged mass size and hormonal status throughout a short follow-up. The risk of malignant transformation seems to be very low also in the presence of a slight increase of mass size. Although patients previously classified as SCS have occasionally been found to have a normal endocrine function at 12 months follow-up, in a recent study, where $75 \mathrm{AI}$ were followed for at least 2 years after diagnosis, the percentage of cases who developed hypercortisolism and had an enlargement of the mass was higher than that reported previously (22). The presence of isolated endocrine abnormalities at diagnosis had predictive value for tumor increase, and a mass size of $3 \mathrm{~cm}$ or more and unilateral radiocholesterol uptake by the tumor had a relevance for the subsequent development of hypercortisolism (22).

Although patients with Cushing's syndrome have clearly established complica- tions, the morbidity of patients with subclinical disease is less clear and controversy exists about the risk of progression from subclinical to overt hypercortisolism. However, recent evidence suggests that SCS or even apparently non-hypersecretory adenoma might also be associated with increased risk for hypertension, insulin resistance state, change in glucose and lipid metabolism, obesity or osteoporosis, in spite of the fact that a clear correlation between hypertension and endocrine abnormalities has not been found $(18,20,22-24)$. In the AI-SIE study, the prevalence of hypertension $(41 \%)$ and diabetes $(10 \%)$ is similar to that reported in the nonhypersecretory adenomas included in the study. On the contrary, there is a slightly significant association between SCS and obesity (38\%) (6).

Nevertheless, weight loss, improvement of hypertension or of glycemic control and modifications of some markers of collagen and bone turnover, similar to those observed in Cushing's syndrome after successful therapy, are frequent findings following unilateral adrenalectomy in AI either with SCS or non-hypersecretory adenomas.

Subtle disturbances of steroid secretion are thus probably present in subjects with $\mathrm{AI}$ in spite of our inability to detect them. Therefore, our challenge is to find a clear marker of endocrine activity in order to identify adrenal masses with autonomous hypercortisolism. A reduction of osteocalcin (BGP), a marker of osteoblastic activity, in patients with AI could be an early marker of subtle hypercortisolism $(18,24)$.

For all of these reasons, we believe that all patients with AI should be screened with an overnight dexamethasone suppression test. Several authors have suggested that a dose higher than the routinely used $1 \mathrm{mg}$ (e.g., 3 $\mathrm{mg}$ ) is indicated to reduce the number of false-positive results. In addition, we believe that cortisol unsuppressibility would be better evaluated by lowering the cut-off to $3 \mu \mathrm{g} /$ $\mathrm{dl}$ rather than the classic $5 \mu \mathrm{g} / \mathrm{dl}$. 
Enhanced 17-OHP response and congenital adrenal hyperplasia. An exaggerated $17-\mathrm{OHP}$ response $(>5 \mathrm{ng} / \mathrm{ml})$ to the ACTH test was probably the most common endocrine alteration observed in patients with AI. The significance of this finding present in 17 to $71 \%$ of cases in different series is still unclear $(5,6)$. In the AI-SIE study, an enhanced 17-OHP response was found in about half of the patients with cortical tumor with no significant difference between unilateral and bilateral or benign and malignant lesions. An enhanced 17-OHP response was also observed in the majority of patients with SCS (68\%). Finally, in our experience, a normalization of this endocrine alteration was observed in the majority of patients who underwent unilateral adrenalectomy.

Although adrenal enlargement associated with an unrecognized congenital adrenal hyperplasia has been well described in homozygous and heterozygous untreated patients, the interpretation of the ACTH test is sometimes controversial $(4,25)$. In fact, an exaggerated 17-OHP response is not specific for 21-OHD and could be merely a sign of disturbed intratumoral steroidogenesis. A recent screening for CYP21 mutations in leucocyte DNA from patients with sporadic adrenocortical tumors failed to detect any mutations $(26,27)$. Furthermore, in a molecular screening for germline 21-OHD performed in about 30 patients with AI and enhanced 17-OHP response to ACTH, we confirmed that 21-OHD is not a major predisposing factor for adrenal tumor development (Mantero F, unpublished data).

Androgen secretion and sex hormoneproducing tumors. Sex hormone-secreting tumors are rare as incidentalomas. Most androgen-secreting neoplasms are adrenocortical carcinomas rather than benign adenomas.

There is controversy over the value of measuring DHEAS $(2,4,5,23,28)$. Because serum DHEAS levels are frequently elevated in patients with clinically manifest adreno- cortical cancer, DHEAS determination could contribute to the differential diagnosis of malignant masses. However, this parameter has low sensitivity with a sub-optimal positive predictive value (PPV). On the other hand, low basal plasma DHEAS levels were frequently observed in patients with SCS and cortical adenomas (24), but the sensitivity and specificity of this parameter were poor (51 and 65\%, respectively, with PPV $10 \%$ ), thus limiting its clinical utility (6). Actually, low DHEAS levels were not clearly associated with the secretory pattern of the masses; the physiological fall of DHEAS with age also has to be kept in mind (21).

Aldosterone-producing adenoma. Primary aldosteronism is an important cause of secondary hypertension and may be significantly more frequent than previously reported (up to $5-15 \%$ of the hypertensive population) and may become the most common form of curable hypertension (29).

The hallmark of primary aldosteronism is hypertension with hypokalemia, suppressed plasma renin activity (PRA) and elevated plasma aldosterone. The absence of hypertension makes aldosteronism an unlikely possibility, although "normotensive primary aldosteronism" has been reported. On the other hand, normokalemia does not exclude aldosteronism since a significant number ( 7 to $38 \%$ ) of patients with aldosteronism are in fact normokalemic (sodium restriction leads to potassium retention, minimizing hypokalemia) (30). Moreover, "normal" aldosterone levels do not exclude the presence of primary aldosteronism, and therefore the combination of an inappropriate aldosterone level and a low PRA is diagnostic both in the presence and in the absence of hypokalemia.

For these reasons, the prevalence of aldosterone-producing adenomas in patients with AI is probably higher than that evaluated from clinical findings, ranging, according to the literature, from 0 to $7 \%$. Moreover, the prevalence of hypertension in patients with 
$\mathrm{AI}$ is higher than in the general population (41 vs 20\%) (6). In the AI-SIE study, aldosterone-producing adenomas were present in only $1.6 \%$ of more than $1000 \mathrm{AI}$. The low prevalence in this study was probably due to the exclusion of cases with severe hypertension and hypokalemia. All patients with aldosteronomas had moderate hypertension and suppressed PRA levels. An apparently normal plasma aldosterone level was found in $30 \%$ of cases. The aldosteronism was normokalemic in $40 \%$ of cases while potassium levels were between $3.5-3.8 \mathrm{mEq} / 1$ in the remaining ones.

One of the major problems in evaluating the renin-angiotensin-aldosterone system is that patients must meet extremely strict criteria in terms of diet (sodium intake), posture, previous and current pharmacological treatment (diuretics, antihypertensive drugs), time of sampling, and evidence of stressful conditions. However, the preferred screening test for primary aldosteronism is the ratio between plasma levels of aldosterone and PRA (31). This ratio is only minimally altered by dietary sodium intake or by most of the concurrent antihypertensive drugs. We advocate measuring serum potassium levels in association with the aldosterone/PRA ratio measured in the upright position (at least $2 \mathrm{~h}$ after the supine position) in all hypertensive patients with an adrenal mass.

An abnormal upright plasma aldosterone (ng/dl)/PRA (ng ml ${ }^{-1} \mathrm{~h}^{-1}$ ) ratio (in our institution $>40$ ) is highly indicative of primary aldosteronism. We believe that a reference aldosterone/PRA ratio should be validated in each center and that the minimal value of PRA included in the ratio should never be lower than the detection limits of the assay. An acute saline suppression test (2 liters of $0.9 \% \mathrm{NaCl}$ solution infused intravenously over a period of $4 \mathrm{~h}$ ) should be performed to confirm the diagnosis. Normally, plasma aldosterone levels decrease to less than $6 \mathrm{ng} /$ $\mathrm{dl}$, whereas subjects with primary aldosteronism fail to show this suppression (30). The fluorohydrocortisone suppression test can be used as an alternative. In the above tests a concomitant value of cortisol should be obtained to distinguish between aldosterone changes due to the test itself from changes due to the circadian rhythm or stress. However, if doubts persist after these tests, bilateral adrenal venous sampling should be performed with evaluation of aldosterone and cortisol levels, especially in cases of bilateral adrenal nodules.

Pheochromocytoma. Although pheochromocytoma is rare $(0.01-0.1 \%$ of hypertensive patients), we strongly recommend that a hormonal screening evaluation be done in all patients to exclude the presence of this potentially lethal condition.

The clinical manifestations of pheochromocytoma are varied in type and severity $(32,33)$. Many patients are totally asymptomatic while some have intermittent symptoms (headache, hypertensive crisis, sweating, cardiac arrhythmias). Hypertension, the hallmark clinical finding, is constant in only about half of the patients, paroxysmal in about one third and absent in approximately one fifth. Some patients do not have hypertensive symptoms and may even be hypotensive, especially in the cases of predominant epinephrine secretion. Several series have reported that up to $76 \%$ of pheochromocytomas are not diagnosed until after death. In the Mayo Clinic experience, the tumor was not suspected in $75 \%$ of 54 autopsied patients with pheochromocytoma, and contributed to death in $55 \%$ of these patients (33).

Clinically silent pheochromocytomas therefore are not rare and their prevalence in patients with AI ranges from 1.5 to $13 \%$ (35). In the AI-SIE study, incidental pheochromocytomas were the second most prevalent form of hyperfunctioning tumors occurring in $4.2 \%$ of all masses. About half of those patients were normotensive, the others had mild to moderate hypertension and none had paroxysmal symptoms of adrenergic discharge (6). Therefore, we strongly recom- 
mend that a hormonal evaluation be done in all patients with AI even if normotensive to exclude the presence of a pheochromocytoma. Measurement of 24-h urinary catecholamine or metanephrine excretion has the highest sensitivity in the diagnosis of pheochromocytoma. Urinary vanillylmandelic acid measurements have a lower sensitivity. Plasma metanephrines may also represent an accurate test for the diagnosis of pheochromocytoma (34). Dynamic tests (glucagon to induce secretion and clonidine as a suppressant) should be reserved for equivocal cases only.

As previously discussed, ${ }^{131} \mathrm{I}$ or ${ }^{123} \mathrm{I}$-MIBG scintigraphy and MRI are the imaging techniques of choice in the suspicion of pheochromocytoma (32). Adrenal scintigraphy with MIBG permits noninvasive, safe, sensitive (sometimes false-negative) and specific (very rare false-positive) localization of pheochromocytoma. At MRI, these tumors usually tend to have bright signal intensity with T2-weighted images. However, we wish to remind the reader that these appearances were not specific: adrenal carcinoma and metastases may present similar hyperintensity on T2-weighted images $(10,11)$.

\section{Management}

Although by definition an incidentaloma is a clinically "silent" mass, a careful investigation including family history and physical examination must be performed. Retrospectively, patients were often found to have had symptoms or signs related to an adrenal tumor. In particular, central obesity, hypertension, diabetes, hirsutism and other symptoms and signs of adrenal hyperfunction should always be considered. Similarly, the presence of extra-adrenal malignancy or associated illnesses such as tuberculosis or familial syndromes should always be considered. In fact, infections occurring in the adrenal gland include fungal and granulomatous infections such as tuberculosis, his- toplasmosis and Pneumocystis carinii pneumonia and usually occurs in immunocompromised patients. We wish to remind the reader that pheochromocytomas can be associated with medullary thyroid carcinoma and hyperparathyroidism (MEN2), von Recklinghausen's disease and the von HippelLindau syndrome (hemangioblastoma of the central nervous system, retinal angioma, renal cysts and carcinoma) (32).

The optimal diagnostic procedure for AI is still controversial and the clinician is called upon to devise a cost-effective approach taking into account the extensive endocrine work-up and radiological investigations that may be necessary (1). In some patients, moreover, the diagnostic process will create considerable anxiety, generate expense, and, if invasive, cause pain and discomfort.

We suggest that a screening evaluation be performed to exclude a functioning adrenal tumor in all patients with AI (Figure 2). The first step should include an overnight low dose dexamethasone suppression test, the measurement of 24-h urinary catecholamines or metanephrines, serum potassium and, in hypertensive patients, upright plasma aldosterone/PRA ratio. DHEAS measurements may show evidence of adrenal androgen excess. Positive results should be followed by specific hormonal evaluations for a definitive diagnosis.

Once a hormone hypersecretion is identified, surgery is indicated. Laparoscopic adrenalectomy has become the method of choice in most centers (35). Although the natural history of SCS and its morbidity are unclear, we advocate adrenalectomy for patients with this condition, especially in the presence of clinical problems (hypertension, obesity, diabetes or osteoporosis) potentially aggravated by glucocorticoid excess. Since the HPA axis and the contralateral adrenal gland may be suppressed by prolonged cortisol secretion, these patients require glucocorticoid therapy both during and following surgery as performed in overt Cushing's syn- 


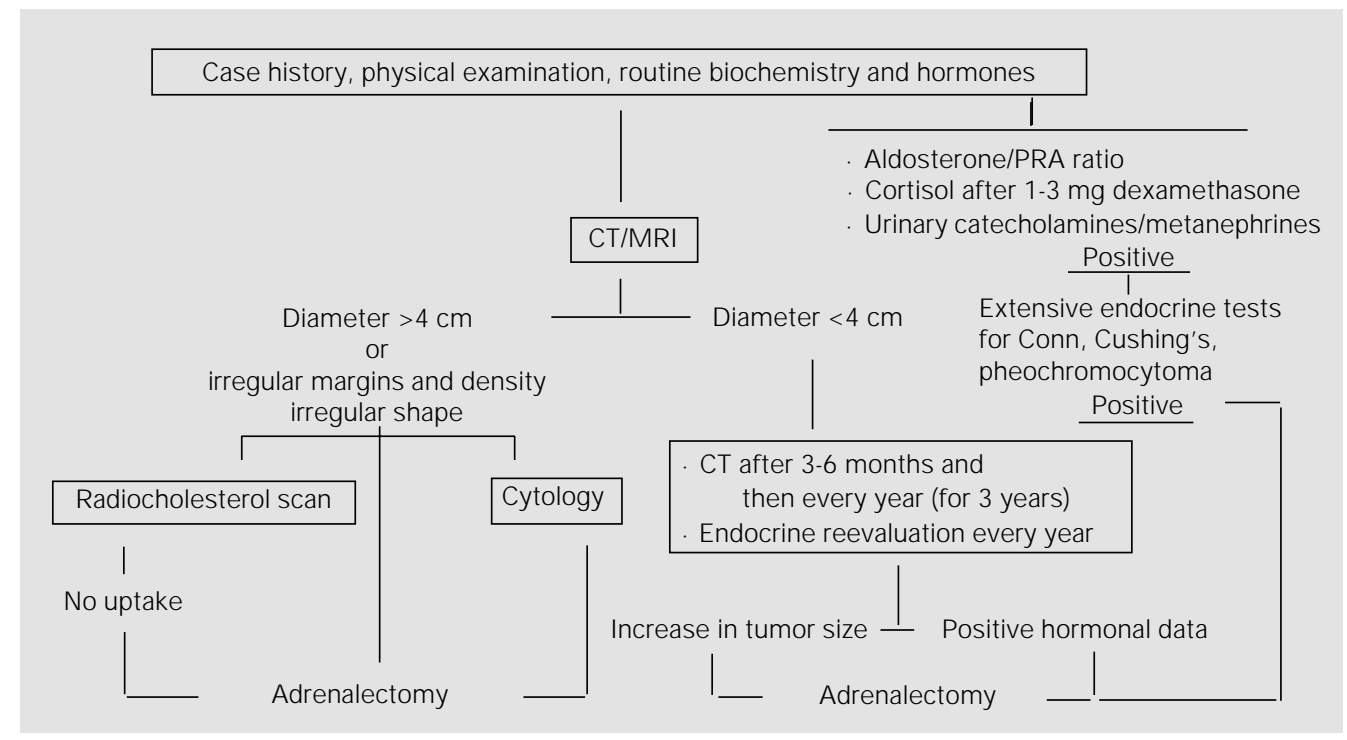

drome. Moreover, we also advocate preoperative medical treatment with an alpha-1 adrenergic antagonist (e.g., prazosin, doxazosin) in patients with incidental pheochromocytoma, even if normotensive. Such treatment will allow expansion of the vascular bed and plasma volume and will reduce the amount of liquids required for maintenance of blood pressure when the tumor is removed.

The size of the AI is a useful parameter to distinguish benign from malignant lesions. Features suggestive of malignancy are large size, irregular margins, non-homogeneous density and an attenuation value $>20 \mathrm{HU}$ on noncontrast-enhanced CT. According to our own experience (see above) a mass larger than $4 \mathrm{~cm}$ should be surgically removed.

In the other lesions, a radiological follow-up (CT/MRI scan and, in unequivocal cases, ultrasound scan) is usually recommended after 6 months. Surgery is indicated for lesions that grow significantly in diameter in the course of follow-up. However, laparoscopy now allows to extend the surgical indication to the removal of relatively small lesions when even a minimal doubt exists about their nature.

In patients with extra-adrenal malignancy, in particular of the lung, the AI (especially if bilateral) may be a metastasis. In this case, an FNA biopsy should be performed.

Finally, adrenal radiocholesterol scintigraphy is a useful imaging test to distinguish benign ("concordant") from malignant ("discordant") masses.

All non-hypersecretory AI smaller than 4 $\mathrm{cm}$ in diameter should be followed up clinically and by serial radiological scanning at regular intervals.

Since malignant masses tend to grow fast, we advocate performing a CT/MRI scan at 3- to 6-month intervals in the first year after the detection of the AI. For the absence of radiation compared to $\mathrm{CT}$, a protocol with MRI and, in unequivocal cases, with ultrasound scan, could be preferable. A clinical (hormonal and radiological) evaluation should be performed after 1 year, then every 1-2 years for a period which awaits to be defined by oncoming data from long-term follow-up of large series.
Figure 2 - Management of adrenal incidentaloma. PRA, Plasma renin activity. 


\section{References}

1. Chidiac RM \& Aron DC (1997). Incidentalomas. A disease of modern technology. Endocrinology and Metabolism Clinics of North America, 26: 233-253.

2. Griffing G (1994). A-I-D-S: The new endocrine epidemic [Editorial comment]. J ournal of Clinical Endocrinology and Metabolism, 79: 1530-1531.

3. Kloos RT, Gross MD, Francis IR, Korobkin M \& Shapiro B (1995). Incidentally discovered adrenal masses. Endocrine Reviews, 16: 460-484.

4. Mantero F \& Amaldi G (1999). Investigation protocol: adrenal enlargement. Clinical Endocrinology, 50: 141-146.

5. Turner HE, Moore NR, Byrne J V \& Wass J AH (1998). Pituitary, adrenal and thyroid incidentalomas. Endocrine-Related Cancer, 5: 131-150.

6. Mantero F, Terzolo M, Amaldi G, Osella G, Masini AM, Alì A, Giovagnetti M, Opocher G \& Angeli A (2000). On behalf of the Study Group on Adrenal Tumors of the Italian Society of Endocrinology. J ournal of Clinical Endocrinology and Metabolism, 85: 637-644.

7. Bornstein SR, Stratakis CA \& Chrousos GP (1999). Adrenocortical tumors: recent advances in basic concepts and clinical management. Annals of Internal Medicine, 130: 759-771.

8. Schulick RD \& Brennan MF (1999). Adrenocortical carcinoma. World J ournal of Urology, 17: 26-34.

9. Kasperlik-Zaluska AA, Rostonowskaa E, Stowinska-Srzednicka J, Midgalska B, J eske W, Mkowska A \& Snochowska H (1997). Incidentally discovered adrenal mass (incidentaloma): investigation and management of 208 patients. Clinical Endocrinology, 46: 29-37.

10. Peppercorn PD, Grossman AB \& Reznek RH (1998). Imaging of incidentally discovered adrenal masses. Clinical Endocrinology, 48: 379-388.

11. Pender SM, Boland GW \& Lee MJ (1998). The incidental nonhyperfunctioning adrenal mass: an imaging algorithm for characterization. Clinical Radiology, 53: 796804.

12. Welch TJ, Sheedy PF, Stephens DH, J ohnson CM \& Swensen SJ (1994). Percutaneous adrenal biopsy: review of a 10year experience. Radiology, 193: 341-344.

13. Silverman SG, Mueller PR, Pinkney LP, Koenker RM \& Seltzer SE (1993). Predictive value of image-guided adrenal biopsy: analysis of results of 101 biopsies. Radiol- ogy, 187: 715-718.

14. Falke THM \& Sandler MP (1994). Classification of silent adrenal masses: time to get practical. J ournal of Nuclear Medicine, 35: 1152-1153.

15. Dwamena BA, Kloos RT, Fendrick MA, Gross MD, Francis IR, Korobkin MT \& Shapiro B (1998). Diagnostic evaluation of the adrenal incidentaloma: decision and cost-effectiveness analyses. J ournal of Nuclear Medicine, 39: 707-712.

16. Bardet S, Rohmer V, Murat A, Guillemot $C$, Marechaud R, Chupin M, Lecomte $P$, Simon D, Delemer B, Schneebelli S, Beutter $D$, J acquin V, Peltier $P$, Charbonnel $B \&$ West France Study Group on Incidentalomas (WFSGI) (1996). 131-I-6ßiodomethylnorcholesterol scintigraphy: an assessment of its role in the investigation of adrenocortical incidentalomas. Clinical Endocrinology, 44: 587-596.

17. Erasmus JJ, Patz J $r$ EF, McAdams HP, Murray J G, Herndon J, Coleman RE \& Goodman PC (1997). Evaluation of adrenal masses in patients with bronchogenic carcinoma using $18 \mathrm{~F}$-fluorodeoxy-glucose positron emission tomography. American J ournal of Roentgenology, 168: 13571360.

18. Ambrosi $B$, Peverelli $S$, Passini $E, \operatorname{Re} T$, Ferrario R, Colombo P, Sartorio A \& Faglia $G$ (1995). Abnormalities of endocrine function in patients with clinically "silent" adrenal masses. European J ournal of Endocrinology, 132: 422-428.

19. Terzolo M, Osella G, Ali A, Borretta G, Cesario F, Paccotti P \& Angeli A (1998). Subclinical Cushing's syndrome in adrenal incidentaloma. Clinical Endocrinology, 48: 89-97.

20. Aron DC (1998). Adrenal incidentalomas and glucocorticoid autonomy (Commentary). Clinical Endocrinology, 49: 157-158.

21. Lavoie H \& Lacroix A (1995). Partially autonomous cortisol secretion by incidentally discovered adrenal adenomas. Trends in Endocrinology and Metabolism, 6: 191-194.

22. Barzon L, Scaroni C, Sonino N, Fallo F, Paoletta A \& Boscaro M (1999). Risk factors and long-term follow-up of adrenal incidentalomas. J ournal of Clinical Endocrinology and Metabolism, 84: 520-526.

23. Osella G, Terzolo M, Borretta G, Magro G, Ali A, Piovesan A, Paccotti P \& Angeli A (1994). Endocrine evaluation of incidentally discovered adrenal masses (incidentalomas). J ournal of Clinical Endocrinol- ogy and Metabolism, 79: 1532-1539.

24. Torlontano M, Chiodini I, Pileri M, Guglielmi G, Cammisa M, Modoni S, Carnevale V, Trischitta V \& Scillitani A (1999). Altered bone mass and turnover in female patients with adrenal incidentaloma: the effect of subclinical hypercortisolism. J ournal of Clinical Endocrinology and Metabolism, 84: 2381-2385.

25. J aresch S, Kornely E, Kley HK \& Schlaghecke R (1992). Adrenal incidentaloma and patients with homozygous or heterozygous congenital adrenal hyperplasia. J ournal of Clinical Endocrinology and Metabolism, 74: 685-689.

26. Kjellman $M$, Holst $M$, Backdahl $M$, Larsson C, Farnebo LO \& Wedell A (1999). No overrepresentation of congenital adrenal hyperplasia in patients with adrenocortical tumours. Clinical Endocrinology, 50: 343-346.

27. Beuschlein $F$, Schulze $E$, Moro $P$, Gensheimer HP, Maser-Gluth C, Allolio B $\&$ Reincke M (1998). Steroid 21-hydroxylase mutations and 21-hydroxylase mRNA expression in human adrenocortical tumors. J ournal of Clinical Endocrinology and Metabolism, 83: 2585-2588.

28. Bencsik Z, Szabolks I, Kovaks Z, Ferencz A, Voros A, Kaszas I, Bor K, Gonczi J, Goth M, Kovacs L, Dohan O \& Szilagyi G (1996). Low dehydroepiandrosterone sulfate (DHEAS) level is not a good predictor of hormonal activity in nonselected patients with incidentally detected adrenal tumours. J ournal of Clinical Endocrinology and Metabolism, 81: 1726-1729.

29. Gordon RD, Klemm SA, Stowasser M, Tunny TJ, Storie WJ \& Rutherford JC (1993). How common is primary aldosteronism? Is it the most frequent cause of curable hypertension? J ournal of Hypertension, 11 (Suppl 5): S310-S311.

30. Biglieri EG, Kater CE \& Mantero F (1995). Adrenocortical forms of human hypertension. In: Laragh J H \& Brenner BM (Editors), Hypertension Pathophysiology, Diagnosis and Management. 2nd edn. Raven Press, New York, 2145-2162.

31. Gordon RD (1995). Primary aldosteronism. J ournal of Endocrinological Investigation, 18: 495-511.

32. Bravo EL (1994). Evolving concepts in the pathophysiology, diagnosis and treatment of pheochromocytoma. Endocrine Reviews, 15: 356-368.

33. Sutton MG, Sheps SG \& Lie JT (1981). Prevalence of clinically unsuspected 
pheochromocytoma: a review of a 50years autopsy series. Mayo Clinic Proceedings, 56: 354-360.

34. Lenders JW, Keiser HR, Goldstein DS, Willemsen BS, Friberg $P$, J acobs MC,
Kloppenborg PW, Thien T \& Eisenhofer G (1995). Plasma metanephrines in the diagnosis of pheochromocytoma. Annals of Internal Medicine, 123: 101-109.

35. Wells SA, Merke DP, Cutler J r GB, Norton
J A \& Lacroix A (1998). The role of laparoscopic surgery in adrenal disease. J ournal of Clinical Endocrinology and Metabolism, 83: 3041-3049. 